\title{
Different Polarity Extracts of Semen Astragali Complanati Wine in Vivo Antioxidant Activity
}

\author{
Xuehui Fan ${ }^{1,2,}$, Qingan Zhang ${ }^{2}$, Chengrui Tian² \\ ${ }^{1}$ School of Physical Education, Shaanxi Normal University, Xi'an, Shaanxi Province, China. \\ ${ }^{2}$ College of Food Engineering and Nutritional Science, Shaanxi Normal University, Xi'an, Shaanxi Province, \\ China. \\ afanxuehui012008@snnu.edu.cn
}

Keywords: semen astragali complanati wine; mice; vivo antioxidant

\begin{abstract}
In this paper, healthy mice are exposed to different polarity extracts of semen astragali complanati wine, ether, ethyl acetate and water through intragastric administration for 30 days. Then the contents of superoxide dismutase (SOD), malonaldehyde (MDA), catalase (CAT), total antioxidant capacity (T-AOC), glutathione (GSH), glutathione peroxidase (GSH-PX) and protein carbonyl in rats' serum are detected, as well as the polyphone contents of different polarity extracts. The results show that semen astragali complanati wine and its different polarity extracts can improve the antioxidant capacity of rats after intragastric administration. Rats from water extracts group have more polyphenol; but their antioxidant capacities are worse than those from ether group and ethyl acetate group. Rats exposed to semen astragali complanati wine have the strongest antioxidant abilities. It indicates that, compared with the low polarity extracts of ether and ethyl acetate, as well as the high polarity extract of water, diversified polarity extracts in the astragali complanati wine can produce synergistic effect, and help rats acquire better antioxidant capacity.
\end{abstract}

\section{Introduction}

Semen Astragali Complanati means the dry, ripe seeds of Astragali complanati R. Br., a kind of perennial dicotyledonous plant. It is a precious traditional Shaanxi "genuine medicinal material" with dual purposes of medicine and food [1]. In this experiment, the direct fermentation method which uses yeast strain is adopted to brew semen astragali complanati wine for the first time. The liquid fermentation process is employed together with dregs fermentation method which combines the advantages of liquid fermentation and solid state fermentation. Compared with existing processing methods of hot salt frying, baking with honey, smoking, steaming with wine and boiling, this fermentation method is carried out under normal temperature and pressure conditions; it can preserve active ingredients of semen astragali complanati. In the biological transformation process, clastic enzyme of microorganism dissolves cell walls, then the cells can leach more effective components; big molecules can be degraded into small molecules which are easily absorbed by human body. Passing through blood-brain barrier, these small molecules play their roles by combining with human body cell protein. But the material basis and mechanism of in vivo antioxidant capacity of astragali complanati wine are not clear. In order to analyze the effective components of astragali complanati wine, this paper evaluates the antioxidant activities of different polarity polyphenol extracts in preliminary, hoping to provide theoretical basis for further research and development of pharmacology.

\section{Experiment Materials and Apparatus}

\subsection{Experiment animal and feed}

Experiment animals are healthy, Kunming ICR male mice, body mass $(24+3) \mathrm{g}$. Animal food 
basically comes from the Experimental Animal Center of Xi'an Jiaotong University.

\subsection{Reagents}

Reagents include semen astragali complanati (purchased from Northwestern Medicine Market, Xi'an, Shaanxi Province), Angel yeast (produced by Angel Yeast Co., Ltd, Yichang, Hubei Province), semen astragali complanati wine (made by Food Processing and Quality Control Laboratory), superoxide dismutase (SOD) test box, malondialdehyde (MDA) test box and catalase (CAT) test box (produced by Nanjing Jiancheng Bioengineering Institute).

\subsection{Apparatus}

Apparatus used in this experiment include AB204-N electronic analytical balance (produced by Mettler Toledo Instruments (Shanghai) Co., Ltd), Multiskan Go ELIASA (produced by Thermo Scientific), TU-1810 UV VIS spectrophotometer (produced by Beijing Persee General Instrument Co., Ltd.) and TGL-16B desk centrifuge (produced by Shanghai Anting Scientific Instrument Factory).

\section{Research Method}

\subsection{The brewing process of semen astragali complanati wine}

Selecting seeds $\rightarrow$ cleaning $\rightarrow$ draining off $\rightarrow$ water immersion and germination $\rightarrow$ drying $\rightarrow$ baking $\rightarrow$ crushing $\rightarrow$ sugar adjusting

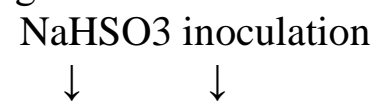

Acidity adjusting $\rightarrow$ sterilization $\rightarrow$ main fermentation (sugar adjusting for 1 time) $\rightarrow$ filtration $\rightarrow$ aging $\rightarrow$ clarification $\rightarrow$ freezing $\rightarrow$ bottling $\rightarrow$ finished products

The optimum fermentation conditions are 26 degrees, $3.7 \mathrm{pH}$ value, $1.1 \%$ yeast addition and 87ppm SO2 addition.

\subsection{Production of different polarity extracts of semen astragali complanati wine}

$50 \mathrm{ml}$ aging astragali complanati wine is added with ether according to the volume ratio of 1:1. After shaking and letting stand until the solution is layered, the upper part of ether extraction is retained. After continuous extraction for 3 times, putting all extractions together, then XAD-7 macroporous resin is used to remove protein and sugar in the solution. Then the solution is put into vacuum rotary evaporation instrument at 40 degree for vacuum concentration to recycle petroleum ether. Ether extract concentrated liquid is obtained. Using $7.4 \%$ ethanol to dissolve the constant volume of $50 \mathrm{ml}$ in a brown flask, the same volume petroleum ether extract is obtained. The remaining solution is processed according to above steps with $50 \mathrm{ml}$ ethyl acetate extraction for 3 times; macroporous resin is used to remove protein and sugar in the solution. After rotary evaporation, the concentrated liquid of ethyl acetate extract is obtained. Using 7.4\% ethanol to dissolve in constant volume of $50 \mathrm{ml}$, the ethyl acetate extract is obtained. The remaining extraction is dissolved with $7.4 \%$ ethanol solution in constant volume of $50 \mathrm{ml}$; the water extract of semen astragali complanati wine is obtained. The extracts are stored in the refrigerator for following experiment.

\subsection{Administration}

Rats with similar weights are randomly divided into 5 groups; each group has 8 rats. The five groups are blank control group (distilled water $0.01 \mathrm{~mL} /(\mathrm{g} \mathrm{bw} \cdot \mathrm{d})$, ether group $(0.01 \mathrm{~mL} /(\mathrm{g} \mathrm{bw} \cdot \mathrm{d})$ ), ethyl acetate group $(0.01 \mathrm{~mL} /(\mathrm{g} \mathrm{bw} \cdot \mathrm{d}))$, water extract group $(0.01 \mathrm{~mL} /(\mathrm{g} \mathrm{bw} \cdot \mathrm{d}))$ and semen astragali complanati wine group $(0.01 \mathrm{~mL} /(\mathrm{g} b \mathrm{~b} \cdot \mathrm{d}))$. One time per day intragastric administration starts after 7 days adaptation feeding, and lasts for 30 days. The daily consumption of food is recorded; the weights of rats are recorded every 7 days. 


\subsection{Animal execution and index detection method}

After 30 days' intragastric administration, water and food are no longer provided. The rats are killed to extract serum at the thirty-first day. The execution of rates go as following: blood is taken out from eyeballs, then cervical vertebra is dislocated to kill the rats immediately; rats' hearts, brains and spleens are weighed and recorded.

Blood serum collection go as following: after weighing, the eyeballs of rats are taken out to collect blood; after blood coagulation for 1 hour, the blood is centrifuged for 10 minus at the speed of 3000 $\mathrm{r} / \mathrm{min}$. The supernatant liquor is taken and conserved to be detected. The T-AOC, SOD, CAT, GSH-Px activity, protein carbonyl content, MDA content and GSH content of serum are measured according to instructions of test boxes.

\subsection{Determination of total phenol content [2-4]}

Gallic acid is used as the standard sample to determine the content. Iml sample solution is diluted with $46 \mathrm{ml}$ distilled water; Iml Folin-Ciocalteu chromogenic agent is added afterwards. After mixing and letting stand for 3 minutes, $3 \mathrm{ml} 2 \%$ sodium carbonate solution is added. Then let the solution stand at room temperature for 2 hours. During every period of time, the mixture is vibrated to realize complete reaction. Then absorbance value is measured at $760 \mathrm{~nm}$. The experiment is repeated for 3 times, and the total phenol content is calculated according to following formula:

Absorbance value $\mathrm{A}=0.0009 \mathrm{x}$ total phenol content (ug) +0.0183

So Folin-Ciocalteu colorimetric method is used to determine the content of total phenol.

\subsection{Data processing}

The data are analyzed by SPSS 19.0 statistical software. All data are represented as $(\mathrm{x} \pm \mathrm{s})$. The significant differences between groups are analyzed through one-way analysis of variance and the SSR method. $\mathrm{P}<0.05$ indicates statistically significant difference.

\section{Experiment Results}

\subsection{The effects of semen astragali complanati wine and its different polarity extracts on rats' food consumption}

As shown in Figure 1, compared with the blank control group, the food consumption conditions of rats from ether group and ethyl acetate group are not significantly different. The food intake of rats from water group and astragali complanati wine group slightly reduce compared with the blank control group.

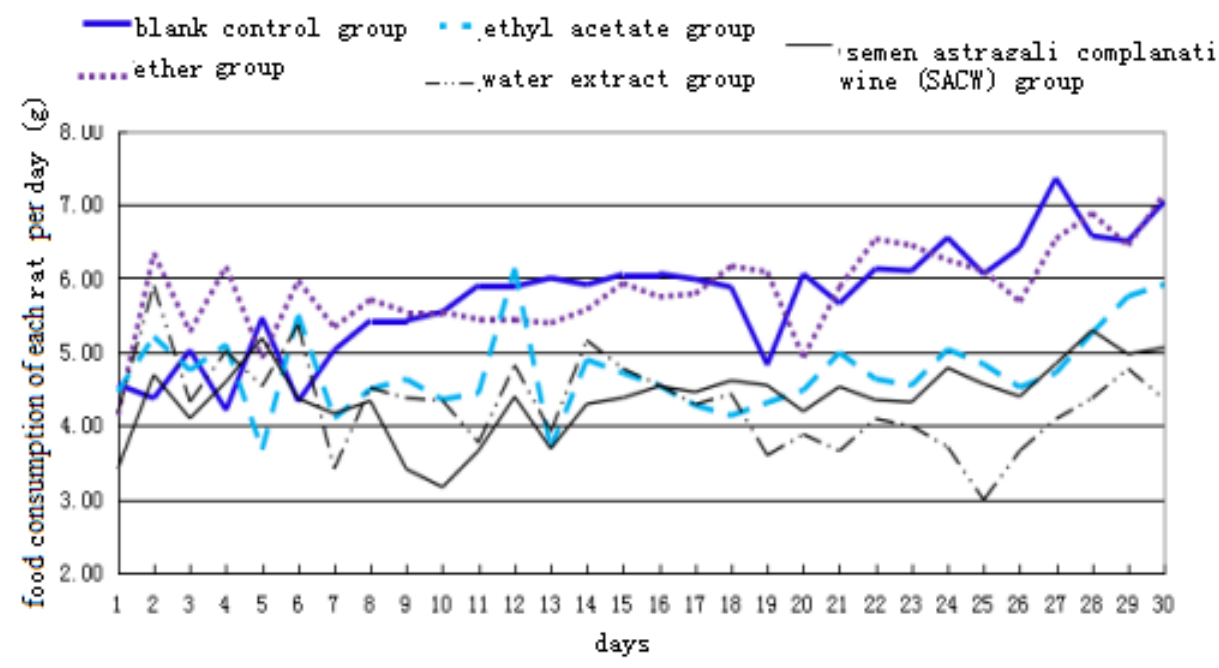

Figure 1. 


\subsection{The effects of semen astragali complanati wine and its different polarity extracts on viscera indexes of rats}

As can be seen from Table 1, semen astragali complanati wine and its different polarity extracts have no significant effects on the heart index of mice. Compared with the control group, kidney indexes of rats from polarity extracts of SACW slightly decrease, but the difference is not significant. The brain indexes of astragali complanati wine group increase. No significant difference is found in the brain indexes of rats from ethyl acetate group, ether group and water extract group. It can be concluded that semen astragali complanati and its various polarity extracts do not damage the organs of mice, and do not have toxic or side effects.

Table 1. Effects of SACW on visceral index (\%)

\begin{tabular}{llll}
\hline Group & $\begin{array}{l}\text { cardiac index } \\
(\%)\end{array}$ & kidney index (\%) & cerebral index (\%) \\
\hline blank control group & $5.72 \pm 0.57$ & $18.34 \pm 1.72$ & $14.31 \pm 2.20$ \\
ether group & $6.08 \pm 0.77$ & $17.70 \pm 1.93$ & $13.76 \pm 1.22$ \\
ethyl acetate group & $5.52 \pm 0.33$ & $17.12 \pm 3.14$ & $14.27 \pm 1.15$ \\
water extract group & $5.56 \pm 0.62$ & $17.74 \pm 2.14$ & $14.34 \pm 2.08$ \\
SACW group & $5.19 \pm 0.36$ & $16.32 \pm 0.28$ & $15.09 \pm 2.19$ \\
\hline
\end{tabular}

Note: different letters indicate the significant differences between different groups (columns). The lowercase letters show significant differences $(\mathrm{P}<0.05)$, while the upper case letters indicate extremely significant differences $(\mathrm{p}<0.01)$; similarly hereinafter.

\subsection{Effects of semen astragali complanati wine and its different polarity extracts on GSH, protein carbonyl, MDA, T-AOC, SOD, CAT and GSH-Px in rats' serum}

From Table 2 and Table 3 it can be found, compared with the blank control group, serum GSH content, T-AOC, SOD, CAT and GSH-Px activity of the rats from ether group significantly increase $(\mathrm{p}<0.05)$; protein carboxyl and MDA content significantly decrease $(\mathrm{p}<0.05)$. For the ethyl acetate group, serum contents of GSH, T-AOC, SOD, CAT and GSH-Px activity significantly increase $(p<0.05)$; while protein carboxyl content significantly decreases $(p<0.05)$; MDA content extremely significant decreases $(\mathrm{p}<0.01)$. For the water extraction group, protein carboxyl content in serum significantly decreases $(\mathrm{p}<0.05)$, SOD content and the activity of GSH-Px significantly increase $(p<0.05)$. Above results indicate that semen astragali complanati wine extracts can decrease in vivo oxidative damage of proteins, increase the activity of oxidase system, protect liver and improve body antioxidant capacity. It can be concluded that semen astragali complanati wine has the function of health care.

Table 2. Effects of SACW and its different polarity extracts on protein carbonyl, T-AOC, MDA contents and SOD activity in rats' serum

\begin{tabular}{llllll}
\hline group & $\begin{array}{l}\mathrm{n} \text { protein carbonyl } \\
(\mathrm{nmol} / \mathrm{ml})\end{array}$ & $\begin{array}{l}\text { T-AOC } \\
(\mathrm{U} / \mathrm{ml})\end{array}$ & $\begin{array}{l}\text { SOD } \\
(\mathrm{U} / \mathrm{ml})\end{array}$ & $\begin{array}{l}\text { MDA } \\
(\mathrm{nmol} / \mathrm{ml})\end{array}$ \\
\hline $\begin{array}{l}\text { blank control } \\
\text { group }\end{array}$ & 8 & $91.18 \pm 8.08$ & $5.28 \pm 0.47$ & $75.17 \pm 4.65$ & $39.63 \pm 1.23$ \\
$\begin{array}{l}\text { ether group } \\
\text { ethyl acetate }\end{array}$ & 8 & $78.01 \pm 17.14^{\mathrm{a}}$ & $9.25 \pm 0.89^{\mathbf{a}}$ & $98.55 \pm 1.42^{\mathbf{a}}$ & $17.81 \pm 1.42^{\mathbf{A}}$ \\
$\begin{array}{l}\text { group } \\
\begin{array}{l}\text { water extract } \\
\text { group }\end{array}\end{array}$ & 8 & $75.79 \pm 10.72^{\mathbf{a}}$ & $8.20 \pm 1.39^{\mathbf{a b}}$ & $92.33 \pm 4.84^{\mathbf{a}}$ & $21.52 \pm 2.36^{\mathbf{a c}}$ \\
SACW group & 8 & $62.61 \pm 18.52^{\mathrm{AbcD}}$ & $11.21 \pm 1.14^{\mathrm{AbcD}}$ & $119.27 \pm 5.69^{\mathbf{A b c d}}$ & $12.17 \pm 3.42^{\mathbf{A b D}}$ \\
\hline
\end{tabular}

Note: different letters indicate the significant differences between different groups (columns). The lowercase letters show significant differences $(\mathrm{P}<0.05)$, while the upper case letters indicate extremely significant differences $(\mathrm{p}<0.01)$; similarly hereinafter. 
Table 3. Effects of SACW and its different polarity extracts on CAT, activity of GSH-Px and GSH content in serum of rats

\begin{tabular}{lclll}
\hline group & $\mathrm{n}$ & $\mathrm{CAT}(\mathrm{U} / \mathrm{ml})$ & $\mathrm{GSH}(\mathrm{mg} / \mathrm{ml})$ & GSH-Px(U/ml) \\
\hline $\begin{array}{l}\text { blank control } \\
\text { group }\end{array}$ & 8 & $51.40 \pm 6.30$ & $1.06 \pm 0.12$ & $1904.83 \pm 72.71$ \\
$\begin{array}{l}\text { ether group } \\
\text { ethyl acetate }\end{array}$ & 8 & $39.24 \pm 7.84^{\mathrm{a}}$ & $2.87 \pm 0.20^{\mathbf{a}}$ & $2244.84 \pm 36.75^{\mathbf{a}}$ \\
$\begin{array}{l}\text { group } \\
\text { water extract }\end{array}$ & 8 & $42.15 \pm 2.37$ & $2.03 \pm 0.08^{\mathbf{a b}}$ & $2205.79 \pm 43.97^{\mathbf{a}}$ \\
group & 8 & $48.33 \pm 4.12$ & $1.10 \pm 0.25^{\mathbf{b}}$ & $2180.42 \pm 52.05^{\mathbf{a}}$ \\
SACW group & 8 & $34.21 \pm 3.52^{\text {abcd }}$ & $3.95 \pm 0.14^{\text {AcD }}$ & $2402.64 \pm 60.47^{\text {abcd }}$ \\
\hline
\end{tabular}

\subsection{Total phenol contents of semen astragali complanati wine and its different polarity extracts}

It can be seen from Table 4, there are differences between the total phenol contents of different polarity extracts. The order from high to low is as following: semen astragali complanati wine group, water extract group, ether group, ethyl acetate group. Except for the semen astragali complanati wine group, the total phenol content is highest in the water extract group $(0.513 \mathrm{mg} \mathrm{mL}-1)$, and lowest in the ethyl acetate group $(0.235 \mathrm{mg} \mathrm{mL}-1)$.

Table 4 Total phenol contents of SACW and its different polarity extracts

\begin{tabular}{ll}
\hline group & Total phenol content/mg $\cdot \mathrm{mL}-1$ \\
\hline ether group & $0.251 \pm 0.023$ \\
ethyl acetate group & $0.235 \pm 0.012$ \\
water extract group & $0.513 \pm 0.056^{\mathrm{ab}}$ \\
SACW group & $0.984 \pm 0.052^{\mathrm{ABc}}$ \\
\hline
\end{tabular}

\section{Analysis and Discussion}

\subsection{Effects of semen astragali complanati wine and its different polarity extracts on the food intake and visceral organs of rats}

Compared with the blank control group, there is no significant difference in the food consumption of rats from the ether group and the ethyl acetate group. The diet of rats from water extract group and semen astragali complanati wine group slightly decrease. It can be seen that different extracts of semen astragali complanati wine have inhibiting effects on the food consumption of mice. However, SACW extracts do not have significant influence on the viscera indexes of rats. Therefore, it can be inferred that in the range of experimental concentration, the inhibiting effects of SACW extracts on diet does not affect their antioxidant protection effects.

\subsection{The relationship between in vivo antioxidant activity of rats and the flavonoid content of semen astragali complanati wine and its different polarity extracts}

The results show that, phenol compounds are found in all extracts of semen astragali complanati wine, and they all show good antioxidant capacities, reducing abilities and scavenging free radicals in serum, indicating that phenol compounds play an important role in the antioxidant capacity of semen astragali complanati wine. As for different extracts, the total phenol content is highest in water extract group $(0.513 \mathrm{mg} \cdot \mathrm{ml}-1)$, and lowest in ethyl acetate group $(0.235 \mathrm{mg} \cdot \mathrm{ml}-1)$, which shows that polyphenol in astragali complanati wine is mainly composed of polarity materials [5-8]. Due to the difference between the micro environment of bio film in which antioxidant and substrate exist and the environment of vitro experiments, the antioxidant activities are greatly different from in vivo and vitro environments. Fat soluble ethyl ether and ethyl acetate extract groups contain less polyphenol than the water extract group, but their anti oxidation abilities are stronger. The reason may be related to the different antioxidant mechanisms of different kinds of polyphenol extracts. 


\subsection{In vivo synergistic antioxidant effects of polyphenol in semen astragali complanati wine and its different polarity extracts}

Experimental results show that both semen astragali complanati wine and its extracts have antioxidant effects, and the antioxidant activity of semen astragali complanati wine is significantly higher than other groups. It indicates synergistic effects between different extracts. These extracts cooperate with each other and improve the in vivo antioxidant effects. This is because, among different extracts of semen astragali complanati wine, mutual coupled oxidation effect and synergist effect can occur in monomers of polyphenol. In addition, two kinds of antioxidants in wine may also produce synergistic antioxidant effects.

\section{Conclusions}

Semen astragali complanati wine is nontoxic. At the same time, the antioxidase activity of semen astragali complanati and its different polarity extracts significantly increase, indicating good antioxidant functions. Antioxidant activity of semen astragali complanati wine is significantly higher than other extraction groups, which shows synergistic antioxidant effects of phenol compounds in wine. In following research, scholars need to pay more attention to analyzing monomer phenol of semen astragali complanati wine through high performance liquid chromatography, and establish the relationship between phenol contents and the antioxidant activity, in order to find out which kind of polyphenol in astragali complanati wine has the direct free radical scavenging effects, establish the dose-response relationship during the process of antioxidase activity improvement, and discover the mechanism, as well as the active components of semen astragali complanati wine.

\section{Acknowledgements}

Fund Project: This paper is supported by the National Natural Science Foundation of China [No.31101324] and Funds for Key Research and Development Programs of Shaanxi Province [No.2017NY-167]. It is also supported by the Basal Research Fund for Central Universities of China [No. GK201404006], the Foundation for Technology Transfer Promotion Projects of Xi'an, Shaanxi Province [No. SXSF003] and the Basic Research Funds for Central Universities [No. GK201602005].

\section{References}

[1] Q.A. Zhang, X.H. Fan, M. Liu, Z.Q. Zhang, Optimal extraction of flavonoid compounds from semen astragali complanati through response surface method and its ability of scavenging DPPH free radical, J. Natural Product Research and Development. 25 (2013).

[2] Lu, Foo, Flavonoid and phenolic glycosides from Salvia officinalis, J. Phytochemistry. 55 (2000).

[3] R.J. Robbins, Phenolicacids in foods: an overview of analytical methodology, J. Agric. Food Chem. 51 (2003).

[4] B.C. Xu, G. Xiao, X.L. Ding, Determination of phenolic acids and proanthocyanidins in tartary buckwheat, J. Food and Fermentation Industries. 28 (2002).

[5] X.X. Cheng, J.S. Yang, F. Z. Ren, Isolation and purification of macroporous resin from total flavonoids of persimmon leaves, J. Hebei Chemical Engineering and Industry. 29 (2006).

[6] Q.A. Hang, X.H. Fan, Z.Q. Zhang, Q. Wang, J. Wang, Antioxidant for phenol extracts from Semen Astragali Complanati, J. Natural Product Research and Development. 24 (2012).

[7] E. Golmakania, A. Mohammadib, T. A. Sanic, H. Kamalib, Phenolic and flavonoid content and antioxidants capacity of pressurized liquid extraction and perculation method from roots of Scutellaria pinnatifida [14] A. Hamilt. subsp alpina (Bornm) Rech. f., J. The Journal of Supercritical Fluids. 95 (2014).

[8] S.C. Liu, J.T. Lin, C.K. Wang, et al., Antioxidant properties of various solvent extracts from lychee (Litchi chinenesis sonn.) flowers, J. Food Chem. 114 (2009). 